\title{
THE LANDSNAIL FAMILY Subulinidae (Gastropoda: Pulmonata) FROM SON LA, VIETNAM, WITH DESCRIPTION OF TWO NEW SPECIES
}

\author{
Do Duc Sang ${ }^{1}$, Do Van Nhuong ${ }^{2}$ \\ ${ }^{1}$ Tay Bac University, *doducsangdhtb@gmail.com \\ ${ }^{2}$ Faculty of Biology, Hanoi National University of Education
}

\begin{abstract}
The paper provided data on the landsnail family Subulinidae in Son La province, northwestern Vietnam. A total of nine species in three genera, viz., Allopeas Baker; 1935; Prosopeas Mörch, 1876 and Tortaxis Pilsbry, 1906 were recorded. Two new species are described, namely Prosopeas muongbuensis sp. n. from Muong Bu commune, Muong La district and Tortaxis comaensis sp. n. from Co Ma commune, Thuan Chau district. Prosopeas muongbuensis sp. $\mathrm{n}$. is diagnosized by a cylindrical, medium-sized, thin, dextral, yellowish-brown shell; whorls convex and separated by an indented, relatively deep suture; callus thick and continued. Tortaxis comaensis sp. $\mathrm{n}$. is recognized by a cylindrical, very large-sized, thick, yellowish-brown shell; whorls flatened and more coarsely ribbed; aperture subovate, oblique, with a thick peristome.
\end{abstract}

Keywords: Subulinidae, new species, landsnail, Son La, Vietnam.

\section{INTRODUCTION}

The pulmonate gastropod family Subulinidae Fischer \& Crosse, 1877 is widely distributed in Central and South America, Africa, Central and South Asia. Not only be found in lands, members of the family are still found in islands in the Pacific and Atlantic Oceans [12, 25]. The family Subulinidae diagnosed by shell's size, specified cylindrical shapes, uniform colors and few decorations. They are one of the most diverse terrestrial gastropods with a wide variety of lifestyles, from ground dwelling species living hidden in the soil to tree climbing, arboreal species living in the canopy, gardens, orchards, residential areas [12].

In Vietnam, seven genera, viz., Opeas Albers, 1850; Allopeas Baker, 1935; Curvella Chaper, 1885; Paropeas Pilsbry, 1906; Prosopeas Mörch, 1876; Subulina Beck, 1837; and Tortaxis Pilsbry, 1906 are already known. Since 1950, however, no any species of the Subulinidae have been added to the list of 22 species previously reported from Vietnam [6, $15,25]$.

Son $\mathrm{La}$ is a province in the northwestern
Vietnam, with a territory of $14,125 \mathrm{~km}^{2}$. Son La topography is deeply differentiated and splitted forming many valleys, basins and alternating mountains with an average elevation of 600-700 $\mathrm{m}$ a.s.l. and variety of different kinds of habitats, e.g. natural forest, limestone mountains, plantation, gardens. Thus, it is believed that Son La will have a rich fauna of landsnails, which are very little known so far. At present, 32 species and subspecies in nine families of pulmonate landsnails are known from Son La [25]. The paper aims to provide faunistic data of subulinid species in Son La, with descriptions of two new species.

\section{MATERIALS AND METHODS}

Specimens were collected by hands from June 2012 to July 2014 in 34 localities from different habitats including limestone forests, caves, limestone karst and residential areas. Material was fixed and preserved in ethanol $70 \%$. Empty shells were preserved in dry storage.

Description format, see Bavay et Dautzenberg (1903, 1908, 1909), Pilsbry (1906), and Pferffer (1861) [2, 3, 4, 22, 23]. Taxonomic terms follow Schileyko (2011) [25]. 
Shell height, shell width, aperture height, aperture width and whorls, were measured for identification.

A total of 2,750 specimens was examined. All specimens are deposited in Soil Organism Research Center (SORC) of Hanoi National University of Education (HNUE), Vietnam National Museum of Nature (VNMN), and Hanoi National University of Education Museum of Biology (HNUEM).

Abbreviations used in this paper are as follows: $\mathrm{H}=$ shell height; $\mathrm{D}=$ shell width; $\mathrm{HA}=$ aperture height; $\mathrm{DA}=$ aperture width; ag=albumin grand; hd=hermaphroditic duct; $\mathrm{uv}=$ oviduct; $\mathrm{sd}=\mathrm{vas}$ deferens; pd=gametolytic sac; $g m=$ multifid gland; v=vagina; $o g=$ atrium; $\mathrm{p}=$ penis; $\quad \mathrm{fl}=$ flagellum; $\quad \mathrm{di}=$ diverticulum; $\mathrm{n}=$ appendix; $\quad \mathrm{ss}=$ sperm $\quad$ sac; $\quad \mathrm{SORC}=$ Soil Organism Research Center; HNUE=Hanoi National University of Education.

\section{RESULTS AND DISCUSSION}

\section{Family Subulinidae Fischer et Crosse, 1877}

Subfamily Subulininae Fischer et Crosse, 1877

Genus Allopeas Baker, 1935

Allopeas Baker, 1935: 84.

Type species: Bulimus gracilis Hutton, 1834.

Allopeas gracile (Hutton, 1834) (Fig. 1A)

Bulimus gracilis Hutton, 1834: 84; Bulimus oparanus - Pfeiffer, 1846: 34; Bulimus oparanus - Reeve, 1848 (1848-1849): pl. 69, fig. 495; Opeas gracilis - Morlet, 1891: 232; Opeas gracile - Möllendorff, 1900: 134; Opeas gracilis - Fischer \& Dautzenberg, 1904: 22; Opeas indicus - Dautzenberg \& Fischer, 1905: 102; Opeas indicus - Ehrmann, 1922: 17; Stenogyra gracilis - Martens, 1867: 83, 375, pl. 19, fig. 5, pl. 22, fig. 13; Allopeas gracile Schileyko, 2011: 9.

Type locality: India, Mirzapur [14].

Material examined: 20 shells (SORC 1401): Bon village, Bon Phang commune, Thuan Chau district $\left(21^{\circ} 23^{\prime} 04.5^{\prime \prime} \mathrm{N}\right.$; $103^{\circ} 46^{\prime 39.1 " E ; ~} 595 \mathrm{~m}$ a.s.1.), limestone mountain, 06.8.2013, coll. Do Duc Sang; 20 shells (SORC 1402): Lech village, Co Noi commune, Mai Son district $\left(21^{\circ} 731^{\prime \prime N}\right.$; $104^{\circ} 9$ '18" E; $685 \mathrm{~m}$ ), limestone mountain, 02.11.2013, coll. Do Duc Sang \& Phung Thi Ngoc.

Diagnosis: Shell subcylindrical, small and yellow. Shell surface with very fine growth lines to nearly smooth. Last whorl accounting for $1 / 2$ shell height, suture deep. Apex acute, aperture ovate, lip simple. Callus thin, transparent. Umbilicus narrow.

Measurements: $\mathrm{H}$ 10.2-13.5 mm, D 3.0-3.5 $\mathrm{mm}, \mathrm{H} / \mathrm{D}$ 3.5-3.9, W 8-9.

Previous records: Bac Giang [8].

Remarks: The species is widely distributed in Asia, Center and South America, and Pacific islands [25]. In Son La, it has been found in disturbed habitats, e.g. gardens, orchards and residential areas.

\section{Genus Prosopeas Mörch, 1876}

Bulimus (Prosopeas) Mörch, 1876: 358.

Type species: Bulimus (Prosopeas) reopstorfi Mörch, 1876.

\section{Prosopeas anceyi Pilsbry, 1906 (Fig. 1B)}

Prosopeas anceyi Pilsbry, 1906: 33, pl. 6, figs. 72, 73, nom. nov. Pro; Prosopeas macilentum - Ancey, 1903 (in Bavay \& Dautzenberg, 1903: 220, pl. 9, figs. 23, 24., which had been pre-occupied; Prosopeas Jaeckel, 1950: 17; Prosopeas anceyi Schileyko, 2011: 10.

Type locality: Vietnam, Bac Kan [23].

Material examined: 20 shells (SORC 1403): Na Loong village, Muong $\mathrm{Bu}$ commune, Muong La district $\left(21^{\circ} 23^{\prime} 28.1^{\prime \prime N}\right.$; $104^{\circ} 02^{\prime} 48^{\prime \prime} \mathrm{E}$; $679 \mathrm{~m}$ ), limestone mountain, 07.8.2012, coll. Do Duc Sang \& Bui Ngoc Cuong; 15 shells (SORC 1404), Na An village, Xuan Nha commune, Van Ho district $\left(20^{\circ} 4359.6^{\prime \prime} \mathrm{N}\right.$; $104^{\circ} 4656$ 'E; $346 \mathrm{~m}$ ), natural forest, 10.11.2013, coll. Do Duc Sang.

Diagnosis: Shell subcylindrical, small and opaque white. Whorls slowly increasing, last whorl accounting for $1 / 2$ shell height. Suture 
shallow. Apex blunt. Aperture conic-ovate. Umbilicus narrow.

Measurements: H 14.5-17.0 mm, D 3.2-3.6 $\mathrm{mm}, \mathrm{H} / \mathrm{D}$ 4.5-4.8, W 9-10.

Previous records: Bac Kan [23].
Remarks: This species is similar to the Prosopeas lavillei, but can be distinguished by the smaller shell, H/D 4.7 (versus 3.5). The species has been known only from Vietnam (Bac Kan) [23].

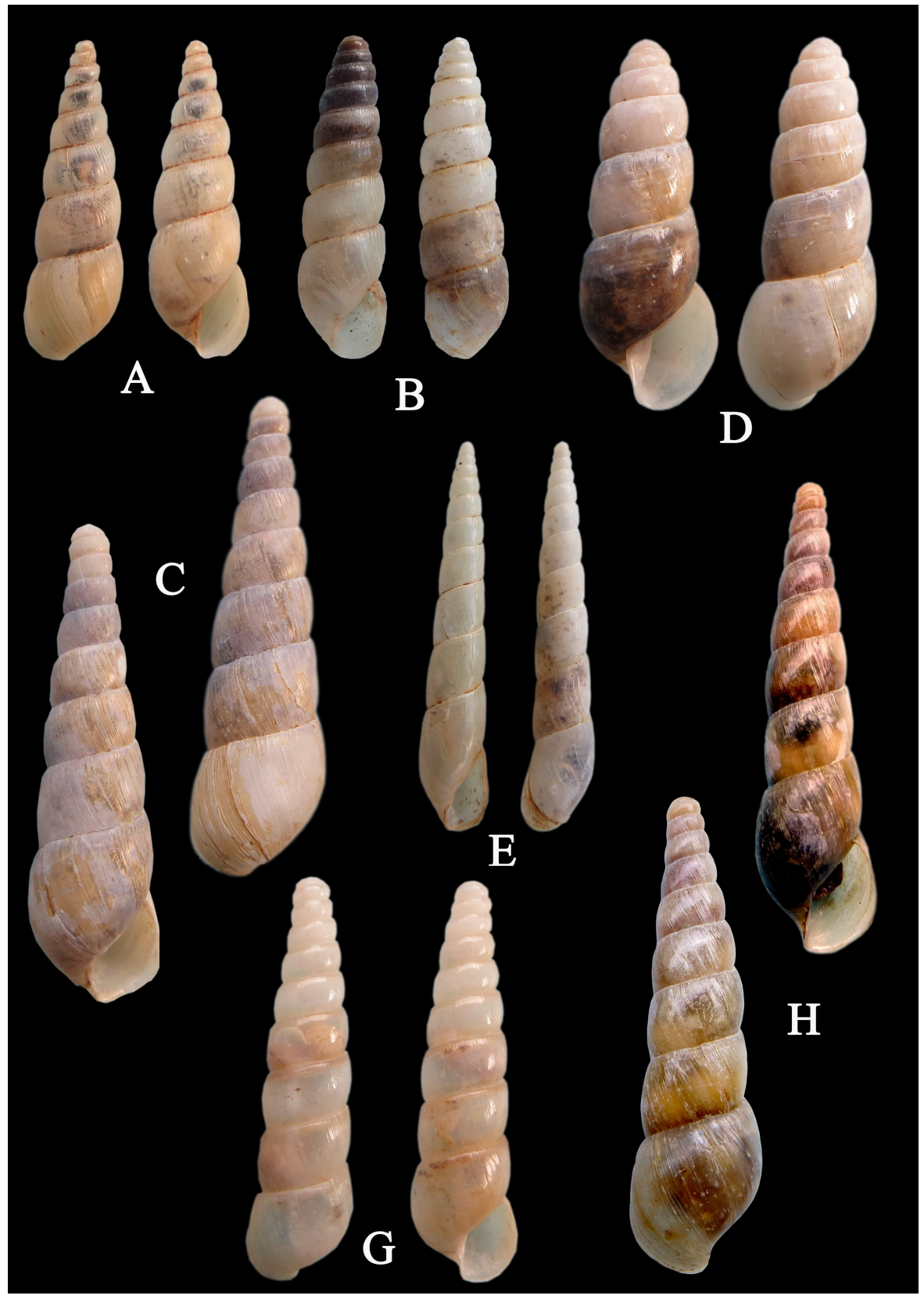

Figure 1. Shell of Subulinidae in Son La

A. Allopeas gracile; B. Prosopeas anceyi; C. Prosopeas excellens; D. Prosopeas ventrosulum; E. Tortaxis elongatissimus; G. Tortaxis lubricus; H. Tortaxis papillosa. 
Prosopeas excellens Bavay et Dautzenberg, 1908 (Fig. 1C)

Prosopeas excellens Bavay \& Dautzenberg, 1908: 247; Prosopeas excellens - Bavay \& Dautzenberg 1909: 182, pl. 10, figs. 11, 12; Prosopeas excellens - Jaeckel, 1950: 17; Prosopeas excellens - Schileyko, 2011: 10.

Type locality: Vietnam, Lai Chau, Phong Tho [3].

Material examined: 15 shells (SORC 1405): Co My village, Chieng Bom commune, Thuan Chau district $\left(21^{\circ} 22^{\prime} 19.8^{\prime \prime} \mathrm{N}\right.$; $103^{\circ} 37^{\prime} 27$ " E; $1,457 \mathrm{~m}$ ), natural forest, 13.06.2013, coll. Do Duc Sang \& Nguyen Thi Huyen; 10 shells (SORC 1406): Pa Cop village, Van Ho commune, Van Ho district $\left(20^{\circ} 45^{\prime} 56^{\prime \prime} \mathrm{N}\right.$; $104^{\circ} 44^{\prime} 30 \mathrm{E} ; 1,013 \mathrm{~m}$ ), limestone mountain, 26.10.2013, coll. Do Duc Sang \& Nguyen Thanh Lap.

Diagnosis: Shell cylindrical, large and pale yellow. Whorls slowly increasing, last whorl accounting for $2 / 5$ shell height. Suture shallow and wide. Shell surface with very fine growth lines. Apex smooth, glossy, more or less rounded. Aperture conic-ovate; parietal callus thin, translucent. Umbilicus narrow.

Measurements: H 38.2-41 mm, D 9.2-10.4 $\mathrm{mm}, \mathrm{H} / \mathrm{D}$ 4.0-4,2, W 9-10.

Previous records: Lai Chau (Phong Tho, Muong Te) [3].

Remarks: This species differs from the Prosopeas lavillei by the larger shell, whorls 910 (versus 11). The species is mainly distributed in limestone forests, and only known from Vietnam (Lai Chau) [3].

Prosopeas ventrosulum Bavay et Dautzenberg, 1908 (Fig. 1D)

Prosopeas ventrosulum Bavay \& Dautzenberg, 1908: 248; Prosopeas ventrosulum - Bavay \& Dautzenberg, 1909: 283, pl. 10, figs. 13, 14; Prosopeas ventrosulum - Jaeckel, 1950: 17; Prosopeas ventrosulum Schileyko, 2011: 10.

Type locality: Vietnam, Lai Chau, Phong Tho [3].
Material examined: 10 shells (SORC 1407): Na Luong village, Chieng Pac commune, Thuan Chau district $\left(21^{\circ} 26^{\prime} 6^{\prime \prime} \mathrm{N} ; 103^{\circ} 45^{\circ} 44.5^{\prime \prime} \mathrm{E} ; 549 \mathrm{~m}\right)$, limestone mountain, 12.5.2014, coll. Do Duc Sang; 15 shells (SORC 1408): Na Ha village, Chieng Mung commune, Mai Son district $\left(21^{\circ} 14^{\prime} 16.3 " \mathrm{~N} ; 104^{\circ} 00{ }^{\prime} 51.7^{\prime \prime} \mathrm{E} ; 667 \mathrm{~m}\right)$, limestone mountain, 16.10.2012, coll. Doan Thi Hoa.

Diagnosis: Shell subcylindrical, medium sized, thickened. Whorls convex and regularly increasing, last whorl large, accounting for $1 / 2$ shell height. Apex blunt, spire elevated. Suture wide. Callus thick. Umbilicus deep and wide.

Measurements: H 15.1-17.5 mm, D 6.0-6.5 $\mathrm{mm}, \mathrm{H} / \mathrm{D}$ 2.5-2.7, W 7. [3].

Previous records: Lai Chau (Phong Tho)

Remarks: This species clearly differs from $P$. anceyi and $P$. excellens by expanded last whorl. It differs from $P$. excellens in having a smooth shell surface and much smaller shell size. The species has been known only from Vietnam (Lai Chau) [3].

\section{Prosopeas muongbuensis Do sp. $\mathbf{n}$.} (Fig. 2. A-C)

Examined material: Holotype (SORC 1415): Nang Phai village, Muong Bu commune, Muong La district, Son La province, Vietnam $\left(21^{\circ} 25^{\prime} 26^{\prime \prime} \mathrm{N}, \quad 104^{\circ} 01^{\prime} 34^{\prime \prime} \mathrm{E}, \quad 711 \mathrm{~m} \quad\right.$ a.s.1. $)$, limestone karst, 12.10.2013, coll. Do Duc Sang. Paratypes (SORC 1416): 20 shells, same data.

Diagnosis: Shell cylindrical, medium-sized, thin, yellowish-brown. Whorls convex and separated by an indented, relatively deep suture; callus thick and continued.

Description: Shell dextral, cylindrical, thin but rather strong, yellowish-corneous, slightly translucent, cylindric-turrited, the apex obtuse. Whorls $6-6 \frac{1}{2}$, quite convex, the apex rounded, rather large, first two whorls smooth, the rest striatulate, the striae a trifle stronger and retraeted near the suture. Suture wide and deep. Aperture small, ovate, slightly oblique, the outer lip thin, arched forward above. Columella concave above, then convex. Callus colour white, thick and continued. Umbilicus very narrow. 
Measurements: Holotype: H $17.2 \mathrm{~mm}, \mathrm{D}$ $6.9 \mathrm{~mm}$, HA $6.3 \mathrm{~mm}$, DA $4.8 \mathrm{~mm}$, W 6 . Paratypes $(\mathrm{n}=20)$ : H: $16.5-19.0 \mathrm{~mm}$, mean 17.9 $\mathrm{mm}$; D: 6.3-7.1 mm, mean $6.7 \mathrm{~mm}$; HA 6.6-7.0 $\mathrm{mm}$; DA 4.6-5.0 mm; W 6-61/2.

Etymology: Named after the type locality, Muong $\mathrm{Bu}$ commune.

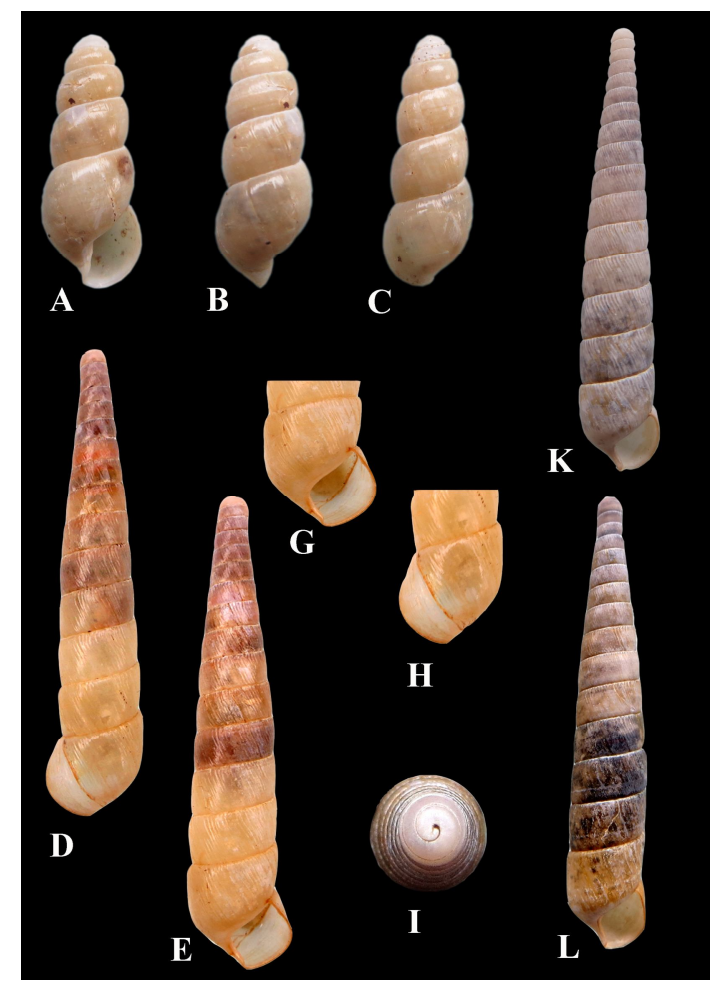

Figure 2. A-C, Prosopeas muongbuensis sp. n.:

Holotype (SORC 1415): A, ventral view; B, lateral view; $\mathrm{C}$, dorsal view;

D-L, Tortaxis comaensis sp. n.: Holotype (SORC 1417): D, dorsal view; E, ventral view;

G, H. last whorl; I. apex; K-L, Paratypes (SORC 1418).

Habitats: The new species has been found in limestone karst area of Son La Province: $\mathrm{Na}$ San plateau; Copia Natural Reserve; Muong Bu commune (type locality); Chieng Bang commune, Quynh Nhai district; Ta Khoa commune, Bac Yen district.

Remarks: A new species is fairly similar to Prosopeas ventrosulum Bavay \& Dautzenberg, 1908 by having shell cylindrical with a blunt apex; teleoconch whorls convex and separated by a deep suture; aperture ovate with the outer lip thin. However, it differs from $P$. ventrosulum from Lai Chau in the lager shell, yellowish-white color, less coarsely sculpture, 6-61/2 (versus 7), the thinner callus, and narrower umbilicus. To date, no living specimens have been found.

\section{Subfamily Rishetiinae Schileyko, 1999 \\ Genus Tortaxis Pilsbry, 1906}

Tortaxis Pilsbry, 1906 (1906-1907): 5.

Type species: Achatina erecta Benson, 1842.

Tortaxis elongatissimus Bavay et Dautzenberg, 1908 (Fig. 1E)

Tortaxis elongatissimus Bavay \& Dautzenberg, 1908: 247; Tortaxis elongatissimus Bavay \& Dautzenberg, 1909: 281, pl. 10, figs. 9, 10; Tortaxis elongatissimus Jaeckel, 1950: 17; Tortaxis elongatissimus Schileyko, 2011: 10.

Type locality: Vietnam, Son La, Van Ho [3].

Material examined: 15 shells (SORC 1409): Pa Kha village, Long Luong commune, Van Ho district $\left(21^{\circ} 477.1^{\prime \prime} \mathrm{N} ; 104^{\circ} 5020\right.$ E; $1.107 \mathrm{~m}$ ), natural forest, 04.7.2014, coll. Do Duc Sang \& Sin Van Thuong; 10 shells (SORC 1410): Ban On cave, Moc Chau district $\left(21^{\circ} 0128.6^{\prime \prime N}\right.$; $\left.103^{\circ} 58^{\prime} 52.2^{\prime \prime} \mathrm{E} ; 895 \mathrm{~m}\right)$, limestone karst, 27.6.2013, coll. Do Duc Sang \& Nguyen Thanh Lap.

Diagnosis: Shell elongate conic, medium sized and pale yellow. Whorls flat and regularly increasing; last whorl large, accounting for $2 / 5$ shell height. Apex acute. Aperture small and triangle; the outer lip thin. Callus thin. Umbilicus very narrow.

Measurements: H 26.2-28.5 mm, D 4.2-4.5 mm, H/D 6.0-6.3, W 13-14.

Previous records: Son $\mathrm{La}$ (Van Ho) [3].

Remarks: This species differs from Tortaxis lubricus and T. mira by having teleoconch whorls more coarsely ribbed; shell width more narrowly developed; suture shallower. It differs from $T$. lubricus by having a weakly shell and more whorls. The species has been known only from Vietnam [3]. 
Tortaxis lubricus Pilsbry, 1906 (Fig. 1G)

Tortaxis lubricus Pilsbry, 1906: 11, pl. 2, figs. 30, 31 .

Tortaxis lubricus Schileyko, 2011: 10.

Type locality: Vietnam, Hai Phong [23].

Material examined: 20 shells (SORC 1411): Na Tre village, Chieng Ban commune, Mai Son district $\left(21^{\circ} 1420^{\prime \prime}\right.$; $103^{\circ} 55^{\prime} 57.3^{\prime \prime E}$; $651 \mathrm{~m}$ ), limestone mountain, 12.9.2013, coll. Do Duc Sang; 20 shells (SORC 1412): Nha Nhung cave, Chieng On commune, Yen Chau district $\left(20^{\circ} 58^{\prime} 42.4^{\prime \prime N} ; 104^{\circ} 10^{\prime} 10.6 " \mathrm{E} ; 802 \mathrm{~m}\right)$, limestone mountain, 26.9.2012, coll. Do Duc Sang \& Bui Ngoc Cuong.

Diagnosis: Shell elongate cylindrical, medium sized and pale brown. Whorls convex and regularly increasing; last whorl large, accounting for $2 / 5$ shell height. Suture deep and wide. Apex blunt. Umbilicus narrow.

Measurements: $\mathrm{H}$ 23.1-25.2 mm, D 5.3-6 $\mathrm{mm}, \mathrm{H} / \mathrm{D}$ 4.2-4.4, W 9.

Previous records: Hai Phong [23].

Remarks: This species is similar to the Tortaxis pilsbry from Bac Kan, but can be distinguished by the smaller shell with light colour, more convex whorls and mostly regularly increasing.

Tortaxis papillosa Dautzenberg et Fischer, 1908 (Fig. 1H)

Tortaxis papillosa Dautzenberg \& Fischer, 1908: 193, pl. 5, figs. 19, 20.

Tortaxis papillosa Schileyko, 2011: 11.

Type locality: Vietnam, Cao Bang, Thach An [8].

Material examined: 20 shells (SORC 1413): Cha May village, Co Ma commune, Thuan Chau district $\left(21^{\circ} 20^{\prime} 46.7^{\prime \prime} \mathrm{N}\right.$; $103^{\circ} 3138.5$ " E; $\left.1,391 \mathrm{~m}\right)$, natural forest, 13.06.2013, coll. Do Duc Sang \& Nguyen Thi Huyen; 10 shells (SORC 1414): Pa Che village, Van Ho commune, Van Ho district $\left(20^{\circ} 47^{\prime} 07.1 " \mathrm{~N} ; \quad 104^{\circ} 45^{\prime} 20.8^{\prime \prime} \mathrm{E} ; \quad 1,050 \mathrm{~m}\right)$, limestone mountain, 26.10.2013, coll. Do Duc Sang \& Nguyen Thanh Lap.

Diagnosis: Shell cylindrically elongate, large sized, glossy and pale yellow. Whorls convex and regularly increasing. Shell surface with fine growth lines to smooth. Suture deep and wide. Apex blunt. Aperture ovate. Umbilicus narrow.

Measurements: H 40.4-44.5 mm, D 9.5$11.1 \mathrm{~mm}, \mathrm{H} / \mathrm{D}$ 4.0-4.3, W 11/2-2-12.

Previous records: Cao Bang (Thach An) [8].

Remarks: The species is similar to Tortaxis pilsbry, but can be distinguished by the larger shell with light colour, more convex whorls. Our specimens differs from original description in having a coarsely rib-striated shell and much larger shell size.

\section{Tortaxis comaensis Do sp. $\mathbf{n}$.}

(Fig. 2.D-L, 3.A-C)

Examined material: Holotype (SORC 1417): Co Ma Commune, Thuan Chau District, Son La Province, Vietnam $\left(21^{\circ} 21^{\prime} 25^{\prime \prime} \mathrm{N}\right.$, $103^{\circ} 31^{\prime 1} 18^{\prime \prime} \mathrm{E}, \quad 1.246 \mathrm{~m}$ a.s.l.), limestone mountain, 08.6.2013, coll. Do Duc Sang. Paratype (SORC 1418): 15 shells, same data.

Diagnosis: Shell cylindrical, very largesized, thickened, yellowish-brown. Whorls flat and more coarsely ribbed; aperture subovate, oblique, with a thick peristome

Description: Shell. Shell very large, dextral, cylindrical, thickened; apex smooth, more or less rounded with inconspicuous growth lines; whorls 16-17 flat becoming increasing regular; shell surface with coarse growth lines which are more rib-like on the more narrowly sculptured, protoconch glossy; colour uniformly yellow, fairly glossy; suture shallow and impressed; aperture subovate, oblique with a simple lip; callus thin and uninterrupted; peristome single, contacting the body whorl and closing the umbilicus.

Genitalia. Oviduct with thin tube, connected between ovary and uterus; penis long, large, proximally cylindrical, and distally forming a swollen globular structure; flagellum long and slender; vas deferens long and thin, entering the epiphallus laterally; vagina long and wide, forming a long, uniform tube with the basally swollen pedunculus; gametolytic sac large, stomach-like; multifid gland with well- 
developed 6 fibers; hermaphroditic duct slender and convoluted; hermaphroditic gland forming multiple clumped alveoli embedded in digestive gland (fig. 3A).

A
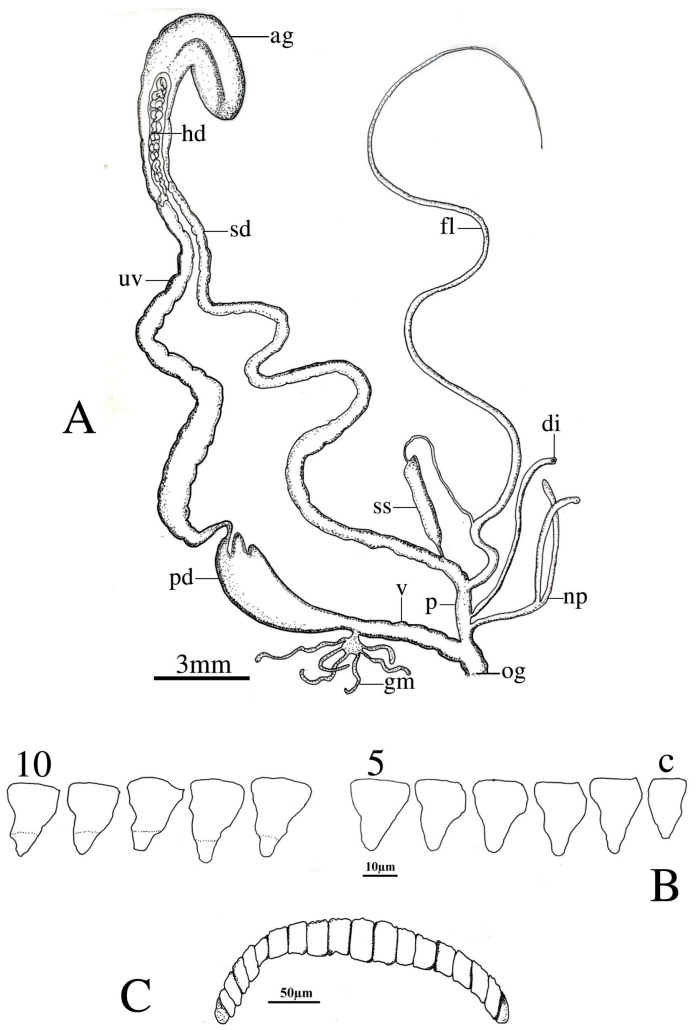

Figure 3. Reproductive system (A), radula (B) and jaw (C) of Tortaxis comaensis sp. $\mathrm{n}$.

Radula. Teeth arranged in line-shaped rows, each row with about 43 teeth $(11: 10: 1: 10$ : 11); A central tooth small, triangle with pointed cusp, latero-marginal teeth arranged in triangle shaped and undifferentiated; marginal teeth size decreasing from the inside out; the jaw significantly less curvature (fig. 4B, 4C).

Measurements. Holotype: H $64.0 \mathrm{~mm}, \mathrm{D}$ $10.2 \mathrm{~mm}$, HA $10.13 \mathrm{~mm}$, DA $6.24 \mathrm{~mm}$; W 17. Paratypes $(n=15)$ : H: 56.1-66.4 mm, mean 60.3 mm; D: 9.8-11.1 mm, mean $10.1 \mathrm{~mm}$; HA 9.5$10.4 \mathrm{~mm}$; DA 6.0-7.3 mm; W 16-17.

Etymology: Named after the type locality, Co Ma commune.

Habitats: New species has been found in limestone forests.
Remarks: This species is most similar to the Tortaxis papillosa Dautzenberg \& Fischer, 1908. However, it is distinguished from T. papillosa by having larger shell with light colour, more whorls (17 versus 12). It also differs from the other Tortaxis species in Vietnam (T. elongatissimus, $T$. erectus, T. lubricus, T. mira, T. permira, T. pilsbry and $T$. servaini) by having larger shell, more whorls.

Acknowledgements: We are grateful to Prof. DSc. Thai Tran Bai (the former director of SORC, HNUE) for kindly providing material and suggesting of this manuscript. We also thank Mr. Nguyen Tho Tue for taking photographs.

\section{REFERENCES}

1. Baker H. B., 1835. Jamaica land snails, 3. Nautilus, 48(3): 83-88.

2. Bavay A., Dautzenberg P., 1903. Description de conquilles nouvelles de 1 Indo-Chine. Journal de Conchyliologie, 51: 201-236.

3. Bavay A., Dautzenberg P., 1908. Molluscorum terrestrium tonkinorum diagnoses. Journal de Conchyliologie, 56: 229-251.

4. Bavay A., Dautzenberg P., 1909. Description de coquilles nouvelles de 1 Indo-Chine. Journal de Conchyliologie, 57: 81-105, 163-206, 279-288.

5. Benson W. H., 1842. Mollusca. In: T. Cantor T. (Ed.). General features of Chusan, with remarks on the flora and fauna of that island. Part 2. Animals observed at Chusan. The Annals and Magazine of Natural History, ser. 1, no. 9: 486-487.

6. Dang N. T., 2008. Overview on the species composition of the landsnails fauna of Vietnam. TAP CHI SINH HOC, 30(4): 1-15.

7. Dautzenberg P., Fischer H., 1905. Liste des mollusques récoltés par M. le Frégate Blaise au Tonkin, et description d'espèces nouvelles. Journal de Conchyliologie, 53: 85-234.

8. Dautzenberg P., Fischer H., 1908. Liste des 
mollusques récoltés par $M$. Mansuy en Indo-Chine et description d'espèces nouvelles. Journal de Conchyliologie, 56: 169-217.

9. Ehrmann P., 1922. Land-und Süßwasserschnecken aus den südl. SchanStaaten, Hinterindien. Sitzungsberichte der naturforschender Gesellschaft zu Leipzig, 45-48: 1-28.

10. Fischer H., 1898. Notes sur la faune du Haut Tonkin. III. Liste des mollusques recueillis par le Dr. A. Billet. Bulletin Scientifique de la France et de la Belgique, 28: 310-338.

11. Fischer H., Dautzenberg P., 1904. Catalogue des mollusques terrestres et fluviatiles de 1'Indo-Chine orientale cites jusqu' à ce jour. In: Mission Pavie, Etudes diverses, III: 161.

12. Fischer P., 1891. Catalogue et distribution gèographique des Mollusques terrestres, fluviatilies \& marins d'une partie de l'IndoChine (Siam, Laos, Cambodge, Cochinchine, Annam, Tonkin). Autun: 1122.

13. Gredler V., 1884. Zur Conchylien-Fauna von China. V. Stück. Jahrbücher der Deutschen Malakozoologische Gesellschaft, 11: 129-161.

14. Hutton T., 1834. On the land shells of India. Journal of the Asiatic Society of Bengal, 3: 81-93.

15. Jaeckel S. H., 1950. Die Mollusken eines tropischen Flußgenistes aus Tonkin. Archiv für Molluslenkunde, 79: 15-20.

16. Mabille J., 1887. Sur quelques mollusques du Tonkin. Bulletin de la Société
Malacologique de France, 4: 73-164.

17. Martens E., 1867. Die Landschnecken. In: Die preussische Expedition nach Ost-Asien. Zoologischer Theil, 2. Berlin. $447 \mathrm{~S}$.

18. Möllendorff O. F., 1900. Zur Binnenmollusken-Fauna von Annams III. Nachrichtsblatt der Deutschen Malakozoologischen Gesellschaft, 32(9-10): 129-139.

19. Mörch O. A. L., 1876. Revision des mollusques terrestres des iles Nicobar. Journal de Conchyliologie, 24: 353-367.

20. Morlet L., 1891. Contribution à la faune malacologique de 1'Indo-Chine. Journal de Conchyliologie, 39: 230-254.

21. Pfeiffer L., 1846. Descriptions of thirty new species of Helicea, belonging to the collection of H. Cuming, Esq. Proceedings of the Zoological Society of London, 14: 28-34.

22. Pfeiffer L., 1861. Descriptions of fortyseven new species of land-shells, from the collection of H. Cuming, Esq. Proceedings of the Zoological Society of London: 20-29.

23. Pilsbry H. A., 1906. Manual of Conchology (2) 18. Achatinidae: Stenogyrinae and Coeliaxinae. Academy of Nature Science, Philadelphia: 1-121.

24. Reeve L., 1848. Conchologia iconica: or, illustrations of the shells of molluscous animals, vol. 5. Monograph of the genus Bulimus. London. Plates I-XLIII, $274 \mathrm{sp}$.

25. Schileyko A. A., 2011. Check list of land pulmonate molluscs of Vietnam (Gastropoda: Stylommatophora). Ruthenica, 21(1): 1-68. 


\title{
HỌ ỐC CẠN Subulinidae (Gastropoda: Pulmonata) Ở SỚN LA VÀ MÔ TẢ HAI LOÀI MỚI
}

\author{
Đỗ Đức Sáng ${ }^{1}$, Đỗ Văn Nhượng ${ }^{2}$ \\ ${ }^{1}$ Trường Đại học Tây Bắc \\ ${ }^{2}$ Trường Đại học Sư phạm Hà Nội
}

\section{TÓM TÁ̀T}

Các loài ốc cạn họ Subulinidae phân bố rộng ở nhiều vùng nhiệt đới trên thế giới, chúng xuất hiện trong các môi trường có độ ẩm cao như rừng trên núi đá vôi, hang động, núi đá vôi, một số còn mở rộng ra khu dân cư, các sinh cảnh nông nghiệp và gây hại cho cây trồng. Tổng cộng đã có 9 loài thuộc 3 giống của Subulinidae được phát hiện ở tỉnh Sơn La. Trong số này, hai loài Prosopeas muongbuensis $\mathrm{sp}$. n. và Tortaxis comaensis sp. n. được mô tả mới cho khoa học; 6 loài, Allopeas gracile, Prosopeas anceyi, P. excellens, P. ventrosulum, Tortaxis lubricus và T. papillosa được bổ sung cho khu hệ ốc cạn tỉnh Sơn La.

Từ khóa: Subulinidae, ốc cạn, loài mới, Sơn La, Việt Nam.

Ngày nhận bài: 14-2-2014 